\title{
An Experiment to Distinguish Between de Broglie-Bohm and Standard Quantum Mechanics
}

\author{
Partha Ghose

\begin{abstract}
S. N. Bose National Centre for Basic Sciences, JD/III Salt Lake, Calcutta 700 098, India
\end{abstract}
An experiment is suggested that is capable of distinguishing between the de Broglie-Bohm theory and standard quantum mechanics.

The proponents of the de Broglie-Bohm quantum theory of motion (dBB) [1-3] have always held that it is constructed to make exactly the same statistical predictions as standard quantum theory (SQT) in every conceivable physical situation. By statistical predictions is meant averages of dynamical variables over all possible "hidden" trajectories at a fixed instant of time (a virtual Gibbs ensemble). The average over a Gibbs ensemble usually turns out to be the same as the average over a time ensemble, i.e., an ensemble built over clearly separated short intervals of time. Although this equivalence holds in SQT, I will show that it breaks down for a special two-particle system in dBB when a certain combination of bosonic and geometrical symmetries holds. It is possible to exploit this feature and do an experiment with a sequence of photon pairs that can distinguish between SQT and dBB, so far thought to be statistically completely equivalent.

To see how predictions of $\mathrm{dBB}$ and SQT can defer in a special case, let us consider a variant of the double-slit experiment in which the wave packets of a pair of identical nonrelativistic particles 1 and 2 are simultaneously diffracted by two slits $A$ and $B$ (which have non-zero widths $d$ much larger than the de Broglie wavelength of the packets, and can be regarded as the sources of the diffracted waves) and overlap in some region $\mathcal{R}$ (see figure) sufficiently far from the slits so that the waves in that region can be regarded as plane waves (the case of Fraunhoffer diffraction). Let us assume that the particles are bosons. The problem is essentially a two dimensional one. So let this plane be the $y-x$ plane, and let us choose the origin $O$ of the coordinate system to be at the mid-point of the line joining 
the two slits $A$ and $B$ as shown in the figure. Then the two-particle wave function in the region $\mathcal{R}$ in the $x-y$ plane can be written as ( [3])

$$
\Psi\left(\vec{r}_{1}, \vec{r}_{2}, t\right)=\sqrt{N} g\left[e^{i\left(\vec{k}_{A} \cdot \vec{r}_{1}+\vec{k}_{B} \cdot \vec{r}_{2}\right)}+e^{i\left(\vec{k}_{A} \cdot \vec{r}_{2}+\vec{k}_{B} \cdot \vec{r}_{1}\right)}\right] e^{-i E t}
$$

where $N$ is a normalization factor, $g$ is a gaussian factor that takes account of the widths of the two slits, and the $k$ 's are wave vectors (with $\left|\vec{k}_{A}\right|=\left|\vec{k}_{B}\right|$ ). Since the double-slit arrangement has a natural symmetry about the line $x=0$, this wave function must be symmetric both with respect to the exchange of the two particles and reflection in the line $x=0$. It is straightforward to show that this wave function can be written in the form

$$
\Psi(\vec{R}, \vec{r})=\sqrt{N} g e^{i \vec{K} \cdot \vec{R}}\left[e^{i \vec{k} \cdot \vec{r}}+e^{-i \vec{k} \cdot \vec{r}}\right]
$$

where

$$
\begin{aligned}
\vec{K} & =\vec{k}_{A}+\vec{k}_{B} \\
\vec{k} & =\vec{k}_{A}-\vec{k}_{B} \\
\vec{R} & =\frac{1}{2}\left(\vec{r}_{1}+\vec{r}_{2}\right) \\
\vec{r} & =\left(\vec{r}_{1}-\vec{r}_{2}\right)
\end{aligned}
$$

The vector $\vec{K}$ is the centre-of-mass momentum of the two-particle system and $\vec{R}$ is the radius vector of the centre-of-mass. The symmetry of the arrangement guarantees that the $x$-component of $\vec{K}$ is zero $\left(K_{x}=0\right.$ ) (and hence $k_{y}=0$ ). (Any $\vec{K}$ and $\vec{k}$ which have these properties are allowed.) It is clear from all this that the relative motion of the particles is decoupled from the centre-of-mass motion. This is a consequence of translation symmetry which holds with plane waves. It is easy to see from (2) that

$$
\begin{aligned}
\Psi^{*} \Psi & =2 g^{2} N[1+\cos 2(\vec{k} \cdot \vec{r})] \\
& \left.=2 g^{2} N\left[1+\cos \frac{2 \pi\left(x_{1}-x_{2}\right)}{L}\right)\right]
\end{aligned}
$$

where $L \approx \lambda / 2 \theta$ is the classical fringe spacing. The probability of joint detection of the particles around points $x(P)$ and $x(Q)$ on a screen with fixed $y$ in the region $\mathcal{R}$ is therefore given by 


$$
P_{12}(x(P), x(Q)) \delta x(P) \delta x(Q)=\int_{x(P)}^{x(P)+\Delta x(P)} d r_{1} \int_{x(Q)}^{x(Q)+\Delta x(Q)} d r_{2}|\Psi|^{2}
$$

which is to be evaluated on a $t=$ constant spatial surface. It contains fourth-order interference terms between 1 and 2 [6], [7]. (The small but finite domains of integration are chosen to take account of the finite size of the detectors.)

So far we have used only standard quantum mechanics (SQT). Let us now introduce the guidance condition of $\mathrm{dBB}$ and calculate the Bohmian velocities of particles 1 and 2 . They are given by

$$
\begin{aligned}
& \vec{v}_{1}=\frac{\vec{j}_{1}}{\Psi^{*} \Psi}=\frac{\hbar}{2 m} \vec{K} \\
& \vec{v}_{2}=\frac{\vec{j}_{2}}{\Psi^{*} \Psi}=\frac{\hbar}{2 m} \vec{K}
\end{aligned}
$$

where $\vec{j}_{1}$ and $\vec{j}_{2}$ are the convection currents of the two particles. It follows from this and the fact that $K_{x}=0$ that $v_{1 x}=v_{2 x}=0$ and so

$$
v_{1 x}+v_{2 x}=0
$$

This implies that

$$
x_{1}(t)+x_{2}(t)=x_{1}(0)+x_{2}(0)
$$

Thus, if the initial positions of the two particles are symmetrical about the line of symmetry $(x=0)$, i.e., if $x_{1}(0)+x_{2}(0)=0$, we must have

$$
x_{1}(t)+x_{2}(t)=0
$$

for all times, i.e., the trajectories will always be symmetrical and parallel about this line. This is the main source of incompatibility between dBB and SQT.

It is important to draw attention to the crucial role played by the combination of bosonic and geometric symmetry in the above argument. Notice that if bosonic symmetry is not imposed on the two-particle wave function (equation (1)), it will not be symmetric under reflection in the line $x=0$. This will mean that with Maxwell-Boltzmann statistics (only one 
term in equation (1)), for example, the trajectories of the two particles can cross each other and the line $x=0$. But the situation is different with bosonic symmetry. This additional symmetry constrains the individual particle trajectories not to touch each other and this line [3]. This brings about a fundamental change - the particle trajectories are clearly separated into two non-intersecting disjoint classes, one above and one below the line of symmetry.

To see what this implies for the two-particle double-slit experiment under consideration, consider the $\mathrm{dBB}$ ensemble to be built up of single pairs of particle trajectories arriving at the screen at different instants of time $t_{i}$ such that the joint probability of detection is given by

$$
\begin{array}{r}
P_{12}=\lim _{N \rightarrow \infty} \sum_{i=1}^{N} \frac{1}{\delta(0)} \int d x_{1} \int d x_{2} P\left(x_{1}, x_{2}, t_{i}\right) \\
\delta\left(x_{1}-x_{1}\left(t_{i}\right)\right) \delta\left(x_{2}-x_{2}\left(t_{i}\right)\right) \delta\left(x_{1}\left(t_{i}\right)+x_{2}\left(t_{i}\right)\right) \\
=\lim _{N \rightarrow \infty} \sum_{i=1}^{N} P\left(x_{1}\left(t_{i}\right),-x_{1}\left(t_{i}\right)\right)=1
\end{array}
$$

where the constraint (9) has been taken into account. Every term in the sum represents only one pair of trajectories arriving at the screen at the points $\left(x_{1}\left(t_{i}\right),-x_{1}\left(t_{i}\right)\right)$ at time $t_{i}$, weighted by the corresponding density $P$. In discrete time ensembles of this type every pair can be separately identified, and it is clear that if the detectors are placed symmetrically about the plane $x=0$, they will record coincidence counts just as predicted by SQT. On the other hand, if they are placed asymmetrically about $x=0$, the joint detection of every pair, and hence also their time average, will produce a null result which is in conflict with the SQT prediction [8].

If all the times $t_{i}$ are put equal to a fixed time $t$ in (10), the sum over $i$ can be converted to an integral over all the trajectories which pass through all the points of the screen at that time (a Gibbs ensemble). Now consider the joint probability of detection around two points $x(P)$ and $x(Q)$ on the screen that are not symmetric about $x=0$. The trajectories that pass through these regions cannot be partners of individual pairs which are constrained by (9), and must therefore belong to different pairs which are not so constrained. If the points are symmetrically situated about $x=0$, the constraint is automatically satisfied. The joint 
probability of detection in this case is therefore given by

$$
\begin{array}{r}
P_{12}(x(P), x(Q), t)=\int_{x(P)}^{x(P)+\Delta x(P)} d x_{1}(t) \int_{x(Q)}^{x(Q)+\Delta x(Q)} d x_{2}(t) \\
P\left(x_{1}(t), x_{2}(t)\right)
\end{array}
$$

which is, in fact, the same as the SQT prediction (5). It becomes impossible therefore to distinguishing between $\mathrm{dBB}$ and SQT using such an experiment, and the trajectories remain "hidden", as correctly maintained by Bohm.

Exactly the same conclusion can be drawn for a pair of photons by using a consistent quantum mechanical formalism for massless relativistic bosons below the threshold of pair creation [9]. In an actual experiment with photons, one must choose identical photons generated by degenerate parametric down-conversion, for example, and make the signal and idler photons pass through the two slits $A$ and $B$ simultaneously. One must also cut down the intensities of the beams to the single pair level. To the best of my knowledge all the conditions necessary to show incompatibility between $\mathrm{dBB}$ and SQT have not been met with in any experiment done so far [6]. Hence the necessity for a critical experiment which can settle the fundamental questions as to whether the wave function description is the most complete possible and also whether the lack of causality in quantum phenomena is really fundamental.

I am grateful to Franco Selleri, Rupa Ghosh, S. M. Roy, C. S. Unnikrishnan, A. S. Majumdar and B. Dutta-Roy for many helpful discussions. I also wish to acknowledge financial support from the Department of Science and Technology, Government of India, through a research grant.

[1] D. Bohm, Phys. Rev. 85, 166, (1952).

[2] D. Bohm and B. J. Hiley, The Undivided Universe (Routlege and Chapman \& Hall, London, 1993). 
[3] P. R. Holland, Quantum Theory of Motion (Cambridge University Press, 1993).

[4] C. R. Leavens, Found. of Physics 25, 229, (1995).

[5] A. Pais, Subtle is the Lord... (Oxford University Press, Oxford, 1982).

[6] R. Ghosh and L. Mandel, Phys. Rev. Letters 59, 1903, (1987).

[7] M. A. Horne, A. Shimony and A. Zeilinger, Phys. Rev. Letters 19, 2209, (1989);

G. Jaeger, M. A. Horne, A. Shimony, Phys. Rev. A 48, 1023, (1993).

[8] Implicit in this is the necessary requirement in $\mathrm{dBB}$ that only particles produce detections and not 'empty' parts of wave functions. See Ref. 3 .

[9] P. Ghose, Found. of Physics 26, 1441, (1996).

FIGURE CAPTION: Two-particle double-slit experiment 


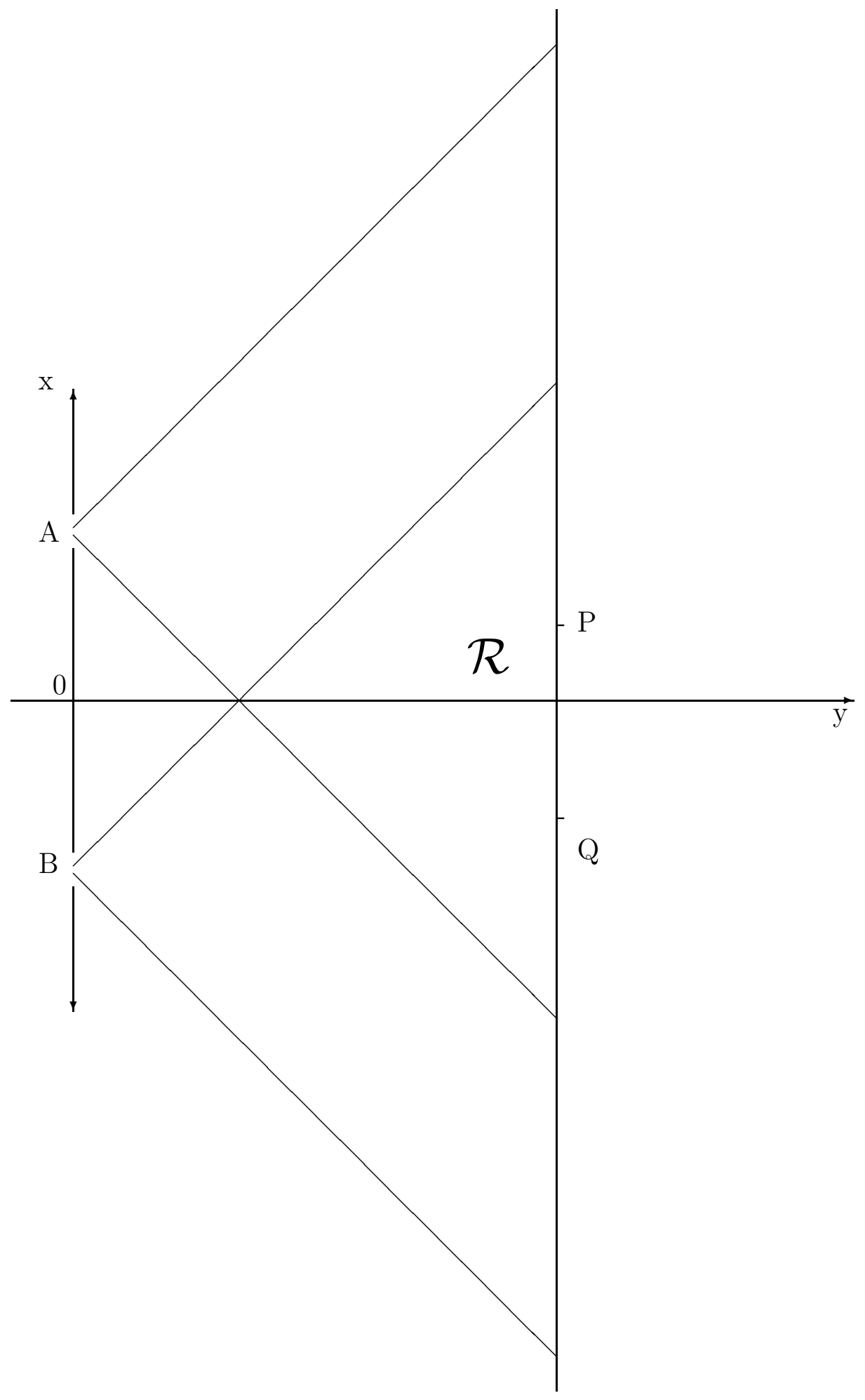

